\title{
Limit theorems for time-dependent averages of nonlinear stochastic heat equations*
}

\author{
Kunwoo Kim \\ Jaeyun Yi \\ POSTECH \\ POSTECH
}

December 14, 2020

\begin{abstract}
We study limit theorems for time-dependent averages of the form $X_{t}:=\frac{1}{2 L(t)} \int_{-L(t)}^{L(t)} u(t, x) d x$, as $t \rightarrow \infty$, where $L(t)=\exp (\lambda t)$ and $u(t, x)$ is the solution to a stochastic heat equation on $\mathbb{R}_{+} \times \mathbb{R}$ driven by space-time white noise with $u_{0}(x)=1$ for all $x \in \mathbb{R}$. We show that for $X_{t}$

(i) the weak law of large numbers holds when $\lambda>\lambda_{1}$,

(ii) the strong law of large numbers holds when $\lambda>\lambda_{2}$,

(iii) the central limit theorem holds when $\lambda>\lambda_{3}$, but fails when $\lambda<\lambda_{4} \leq \lambda_{3}$,

(iv) the quantitative central limit theorem holds when $\lambda>\lambda_{5}$,

where $\lambda_{i}$ 's are positive constants depending on the moment Lyapunov exponents of $u(t, x)$.
\end{abstract}

Keywords: Stochastic heat equation, weak law of large numbers, strong law of large numbers, central limit theorem

MSC 2020 subject classification: 60H15, 60F 15, 60F05

\section{Introduction and main results}

We consider the one-dimensional nonlinear stochastic heat equation

$$
\left\{\begin{array}{l}
\frac{\partial}{\partial t} u(t, x)=\frac{1}{2} \frac{\partial^{2}}{\partial x^{2}} u(t, x)+\sigma(u(t, x)) \dot{W}(t, x), \quad t>0, x \in \mathbb{R}, \\
u(0, x)=1, \quad x \in \mathbb{R},
\end{array}\right.
$$

where $\sigma: \mathbb{R} \rightarrow \mathbb{R}$ is a Lipschitz continuous function with the Lipschitz constant $\operatorname{Lip}_{\sigma}>0$ and $\dot{W}$ is a space-time Gaussian white noise. Under these conditions, it is well known that (1.1) admits the unique random field solution (see e.g. [26]) given by

$$
u(t, x)=1+\int_{(0, t) \times \mathbb{R}} p_{t-s}(y-x) \sigma(u(s, y)) W(d s d y), \quad \text { for } t>0, x \in \mathbb{R},
$$

* Research supported by the NRF (National Research Foundation of Korea) grants 2019R1A5A1028324 and 2020R1A2C4002077. 
where $p_{t}(x):=(2 \pi t)^{-1 / 2} \exp \left(-x^{2} / 2 t\right)$ for $t>0$ and $x \in \mathbb{R}$. Since $u_{0}(x)=1$ for all $x \in \mathbb{R}$, it is easy to see that $u(t, x)$ is stationary in $x$ (see e.g. [12]). We further assume that $\sigma$ satisfies

$$
\sigma(0)=0 \quad \text { and } \quad \mathrm{L}_{\sigma}:=\inf _{w \in \mathbb{R}}\left|\frac{\sigma(w)}{w}\right|>0,
$$

which guarantees that $u(t, x)>0$ for all $t \geq 0$ and $x \in \mathbb{R}$ with probability 1 (see e.g. [6,23]) and

$$
\mathrm{L}_{\sigma}^{2} w^{2} \leq \sigma^{2}(w) \leq \operatorname{Lip}_{\sigma}^{2} w^{2} \quad \text { for every } w \in \mathbb{R} .
$$

Thanks to (1.3), we also have the following (see e.g. $[15,20]$ ):

$$
0<\underline{\gamma}(p) \leq \bar{\gamma}(p)<\infty \quad \text { for all } p \geq 1
$$

where $\bar{\gamma}(p)$ and $\underline{\gamma}(p)$ are the upper and lower $p$-th moment Lyapunov exponents respectively defined as

$$
\begin{aligned}
& \bar{\gamma}(p):=\limsup _{t \rightarrow \infty} \frac{1}{t} \log \mathbb{E}\left[|u(t, 0)|^{p}\right], \\
& \underline{\gamma}(p):=\liminf _{t \rightarrow \infty} \frac{1}{t} \log \mathbb{E}\left[|u(t, 0)|^{p}\right] .
\end{aligned}
$$

In addition, $\bar{\gamma}(1)=\gamma(1)=0$ since $\mathbb{E}[u(t, x)]=1$ and $p \mapsto \bar{\gamma}(p)$ is a convex function, which implies that $\bar{\gamma}$ has a right derivative (see $[15,20]$ ).

The main objective of this paper is to study limit theorems for time-dependent averages of the form

$$
\frac{1}{\left|\Lambda_{L(t)}\right|} \int_{\Lambda_{L(t)}} u(t, x) d x \quad \text { as } t \rightarrow \infty,
$$

where $\Lambda_{L(t)}:=\{x \in \mathbb{R}:|x| \leq L(t)\}$ with $L(t):=e^{\lambda t}$ for fixed constant $\lambda>0$. Here are the main theorems:

Theorem 1.1. (The Weak Law of Large Numbers) If $\lambda>\bar{\gamma}^{\prime}(1)$, then for every $\epsilon>0$,

$$
\lim _{t \rightarrow \infty} \mathbb{P}\left\{\left|\frac{1}{\left|\Lambda_{L(t)}\right|} \int_{\Lambda_{L(t)}}(u(t, x)-1) d x\right| \geq \epsilon\right\}=0,
$$

where $\bar{\gamma}^{\prime}$ is defined as the right derivative of $\bar{\gamma}$.

Theorem 1.2. (The Strong Law of Large Numbers) If $\lambda>\frac{5 \bar{\gamma}(4)}{6}$, then

$$
\frac{1}{\left|\Lambda_{L(t)}\right|} \int_{\Lambda_{L(t)}} u(t, x) d x \rightarrow 1, \quad \text { as } t \rightarrow \infty \text {, a.s.. }
$$

Theorem 1.3. (The Criteria for the Central Limit Theorem) Let us define

$$
F_{\lambda}(t):=\frac{\int_{\Lambda_{L(t)}}(u(t, x)-1) d x}{\sqrt{\operatorname{Var}\left(\int_{\Lambda_{L(t)}} u(t, x) d x\right)}} .
$$

Then as $t \rightarrow \infty$ : 
(i) $F_{\lambda}(t) \stackrel{\mathcal{L}}{\rightarrow} N(0,1)$, if $\lambda>\inf _{0<\epsilon<1}\left\{\frac{2(\bar{\gamma}(2+\epsilon)-\gamma(2))}{\epsilon}-\underline{\gamma}(2)\right\}$;

(ii) $F_{\lambda}(t) \rightarrow 0$ in probability, if $\lambda<\sup _{0<\epsilon<1}\left\{\frac{2(\underline{\gamma(2)-\bar{\gamma}(2-\epsilon))}}{\epsilon}-\underline{\gamma}(2)\right\}$,

where $N(0,1)$ denotes the standard normal distribution.

Remark 1.4. Since $\bar{\gamma}(p)$ is a convex function for $p \geq 1$ and $\bar{\gamma}(p) \geq \underline{\gamma}(p)$ for all $p \geq 1$, we have

$$
\inf _{0<\epsilon<1}\left\{\frac{2(\bar{\gamma}(2+\epsilon)-\underline{\gamma}(2))}{\epsilon}-\underline{\gamma}(2)\right\} \geq \sup _{0<\epsilon<1}\left\{\frac{2(\underline{\gamma}(2)-\bar{\gamma}(2-\epsilon))}{\epsilon}-\underline{\gamma}(2)\right\} .
$$

Theorem 1.5. (The Quantitative Central limit Theorem) Suppose that

$$
\lambda>2^{9} \operatorname{Lip}_{\sigma}^{4}+\bar{\gamma}(4)-2 \underline{\gamma}(2) .
$$

Let $d_{T V}$ denote the total variation distance and $Z \sim N(0,1)$. Then there exists a positive constant $C=C(\lambda, \sigma)$, which is independent of $t$, such that

$$
d_{T V}\left(F_{\lambda}(t), Z\right) \leq C e^{-C t},
$$

for all sufficiently large $t$.

We first note that the above main theorems are related to intermittency in the sense of Carmona and Molchanov [2]. In order to explain this, we assume $\gamma(p):=\bar{\gamma}(p)=\gamma(p)$ for all $p>0$. Then, intermittency is defined in terms of $\gamma(p)$. More precisely, a random field $\{u(t, x) ; t \geq 0, x \in \mathbb{R}\}$ is called fully intermittent if

$$
\frac{\gamma(p)}{p}<\frac{\gamma(p+1)}{p+1} \text { for all } p \geq 1
$$

This full intermittency condition, along with the ergodicity of the random field, implies that the random field develops many different tall peaks over small regions (intermittent islands) separated by large areas (voids) (see [2,3,20]; see also [21,22] which characterize the geometric structure of the tall peaks). Here, the condition (1.10) tells us the relation between the sizes of the intervals on which Theorems 1.1 and 1.3 hold. In other words, if the solution $u(t, x)$ to (1.1) is fully intermittent and $\gamma(p):=\bar{\gamma}(p)=\underline{\gamma}(p),(1.10)$ implies that

$$
\begin{aligned}
\lambda_{*}:=\inf _{0<\epsilon<1}\left\{\frac{2(\gamma(2+\epsilon)-\gamma(2))}{\epsilon}-\gamma(2)\right\} & =\lim _{\epsilon \downarrow 0}\left\{\frac{2(\gamma(2+\epsilon)-\gamma(2))}{\epsilon}-\gamma(2)\right\} \\
& =2 \gamma^{\prime}(2)-\gamma(2) \\
& =\lim _{\epsilon \downarrow 0}\left\{\frac{2(\gamma(2)-\gamma(2-\epsilon))}{\epsilon}-\gamma(2)\right\} \\
& =\sup _{0<\epsilon<1}\left\{\frac{2(\gamma(2)-\gamma(2-\epsilon))}{\epsilon}-\gamma(2)\right\} .
\end{aligned}
$$

Thus, the central limit theorem (Theorem 1.3) holds if $\lambda>\lambda_{*}$ and fails if $\lambda<\lambda_{*}$. We can also compare Theorem 1.3 to Theorem 1.1. If $\gamma$ is differentiable twice,

$$
\frac{d}{d p}\left(p \gamma^{\prime}(p)-\gamma(p)\right)=p \gamma^{\prime \prime}(p) \geq 0,1
$$

\footnotetext{
${ }^{1}$ Note that $(1.10)$ does not guarantee $\gamma^{\prime \prime}(p)>0$. The counterexample would be the case where a random variable has the $p$-th moment Lyapunov exponent as $\gamma(p)=p-1$ for $p \geq 1$.
} 
since $\gamma$ is convex. Thus, we have

$$
2 \gamma^{\prime}(2)-\gamma(2) \geq \gamma^{\prime}(1)
$$

This says that the central limit theorem (Theorem 1.3) requires a bigger interval $\Lambda_{L(t)}$ than the weak law of large numbers (Theorem 1.1) if $\bar{\gamma}(p)=\underline{\gamma}(p)$ and $u(t, x)$ is fully intermittent. Indeed, when $\sigma(w)=w$ (in this case, (1.1) is called the parabolic Anderson model), Ghosal and Lin [17] recently showed that $\gamma(p):=\bar{\gamma}(p)=\underline{\gamma}(p)=\frac{p\left(p^{2}-1\right)}{24}$ for all $p>0$ (see also [8] for positive integer valued $p$ and [13] for delta initial data). Therefore, for the parabolic Anderson model, we can find how large the interval should be in order for Theorems 1.1-1.5 to hold. For the general $\sigma$, one may use the moment comparison principle (see $[7,19]$ ) to get the bounds on $\underline{\gamma}$ and $\bar{\gamma}$.

Theorems 1.1 and 1.2 imply that

$$
\int_{\Lambda_{L(t)}} u(t, x) d x \asymp \mathbb{E}\left[\int_{\Lambda_{L(t)}} u(t, x) d x\right]=2 L(t)=2 e^{\lambda t},
$$

and Lemma 5.1 says that

$$
\operatorname{Var}\left(\int_{\Lambda_{L(t)}} u(t, x) d x\right) \asymp L(t) e^{\gamma(2) t}=e^{(\lambda+\gamma(2)) t} .
$$

On the other hand, if we consider (1.1) with $\sigma(w)=1$ for all $w \in \mathbb{R}$ and call the solution $Z(t, x)$, i.e.,

$$
Z(t, x)=1+\int_{(0, t) \times \mathbb{R}} p_{t-s}(y-x) W(d s d y),
$$

then it is easy to see that $\int_{\Lambda_{L(t)}} Z(t, x) d x$ is a Gaussian random variable with mean $2 L(t)$ and

$$
\operatorname{Var}\left(\int_{\Lambda_{L(t)}} Z(t, x) d x\right) \asymp L(t) .
$$

Therefore, the central limit theorems (Theorems 1.3 and 1.5) may provide a quantification that the tall peaks in the parabolic Anderson model can occur with a higher probability than the tall peaks of the same height for $Z(t, x)$.

Limit theorems for time-dependent averages of random fields have been mostly studied for random fields on $\mathbb{Z}^{d}$ (see $[1,10,16]$ to only name a few). In particular, Cranston and Molchanov in [10] considered the parabolic Anderson model on $\mathbb{Z}^{d}$ and obtained the weak law of large numbers and the central limit theorem that correspond to Theorems 1.1 and 1.3 (i) (Theorem 1.3 (ii) did not appear in [10]). They also showed quenched asymptotics (i.e., $t^{-1} \log \sum_{x \in \Lambda_{L(t)}} u(t, x) \rightarrow \tilde{\gamma}$ a.s. where $\tilde{\gamma}$ is the almost sure Lyapunov exponent) if $L(t)$ grows slower than exponentially, and found the upper bound for the quenched asymptotics when $\lambda$ is small (they called it Transition Range). Those quenched asymptotics and transition range result were simply obtained from the known result about the almost sure Lyapunov exponent $([11])$ and the additivity of the time-dependent average (i.e., $\sum_{x \in \Lambda_{L(t)}} u(t, x)=$ $\left.u\left(t, x_{1}\right)+\cdots+u\left(t, x_{L(t)}\right)\right)$.

On the other hand, when the spatial space is $\mathbb{R}^{d}$, most of the work has been done recently for quantitative central limit theorems for spatial averages of the form $\int_{[-R, R]^{d}} g(u(t, x)) d x$ as 
$R \rightarrow \infty$ for fixed $t>0$ by beautifully using the Malliavin calculus and Stein's method where $g: \mathbb{R} \rightarrow \mathbb{R}$ is a function such that $g(u)=u$ (see [18]), $g$ is a globally Lipschitz function (see [4]), or $g$ is a locally Lipschitz function (see [5]). The only literature which mentions about the timedependent average that we know is the recent one by Chen et al in [5] where they show the quantitative central limit theorem for time-dependent averages of the form $\int_{[-N, N]^{d}} u\left(t_{N}, x\right) d x$ when $t_{N}=o(\log N)$ as $N \rightarrow \infty$ (see [5, Corollary 2.7]). However, a precise box size was not given there. As far as the strong law of large numbers of the form (1.7) is concerned, to the best of our knowledge, there have been no results even for the parabolic Anderson model on $\mathbb{Z}^{d}$ and Theorem 1.2 is new. On the other hand, when $L(t)$ is small or $\lambda$ is small, we were not able to obtain the quenched asymptotics either the transition range result as in [10] since we do not have the additivity of the average, and this could be considered as future work. Instead, we show that the central limit theorem fails when $\lambda$ is small (see Theorem 1.3 (ii)), which was not considered in $[5,10]$.

We now highlight some main ideas of the proofs of the limit theorems above. For Theorems 1.1 and 1.3, we use the von Bahr-Esseen inequalities and Lyapunov's criterion as in [10]. To use those, one needs to produce independent random variables which are close to the solution to (1.1). In [10], Cranston and Molchanov used the Feynman-Kac formula for the solution to produce independent random variables. However, there is no formal Feynman-Kac formula (as in [10]) for the solution to (1.1) (there is a renormalized Feynman-Kac formula with Wick exponential, but it may not be useful here). Thus, we use the localization argument developed by Conus et al in [9] and further quantified by Khoshnevisan et al in [22]. By the localization argument, we can see that the solution is localized so that whenever $x$ and $y$ are far apart, depending on time variable $t, u(t, x)$ and $u(t, y)$ are almost independent (see Lemma 2.3). We also note that along with the localization argument, we use a quantitative form of the Kolmogorov continuity theorem in an elegant way to show Theorem 1.2 (see Lemma 4.2). Indeed, our methods can be applied to a more general setting such as time-dependent averages of the form $\frac{1}{\left|\Lambda_{L(t)}\right|} \int_{\Lambda_{L(t)}} g(u(t, x)) d x$ for some function $g$. For example, by following the proofs of Theorems 1.1 and 1.2 , one can easily show that Theorems 1.1 and 1.2 holds when $g: \mathbb{R} \rightarrow \mathbb{R}$ is a globally Lipschitz function. That is, one may show that

$$
\frac{1}{\left|\Lambda_{L(t)}\right|} \int_{\Lambda(t)}\{g(u(t, x))-\mathbb{E}[g(u(t, x))]\} d x
$$

converges to 0 in probability or almost surely whenever $L(t)$ satisfies the assumptions in Theorems 1.1 and 1.2 (we leave this for the interested reader). Lastly, for Theorem 1.5, we follow the method of [18], which combines the Malliavin calculus and Stein's method.

The rest of the paper is organized as follows. In Section 2, we introduce some lemmas and partitions of an interval used for the proofs of Theorems 1.1-1.3. In the following sections (Sections 3-6), we provide the proofs of Theorems 1.1-1.5 respectively.

\section{Preliminaries}

In this section, we introduce some lemmas and partitions of the interval $[-L, L]$ which play a significant role on the proofs of the main theorems. 


\subsection{Some important lemmas}

The following lemma is the von Bahr-Esseen inequality which will be used in the proof of Theorem 1.1.

Lemma 2.1 (von Bahr-Esseen inequality [25]). Let $\left\{X_{n}\right\}_{n \in \mathbb{Z}^{+}}$be a sequence of independent mean zero random variables, and $1 \leq r \leq 2$. Then

$$
\mathbb{E}\left[\left|\sum_{i=1}^{n} X_{i}\right|^{r}\right] \leq 2 \mathbb{E}\left[\sum_{i=1}^{n}\left|X_{i}\right|^{r}\right]
$$

for all integers $n \geq 1$.

We note that if we just use Jensen's inequality, we get $n^{r-1}$ which is greater than 2 for large $n$ in the von Bahr-Esseen inequality. In other words, the von Bahr-Esseen inequality gives a better bound than Jensen's inequality and it plays an essential role in the proof of Theorem 1.1. The following lemma is the (generalized) von Barh-Esseen inequality when $r \geq 2$ and this inequality also provides a better bound than Jensen's inequality. We will use this inequality for the proof of Theorem 1.2.

Lemma 2.2 (Generalized von Bahr-Esseen inequality [14]). Let $\left\{X_{n}\right\}_{n \in \mathbb{Z}^{+}}$be a sequance of independent mean zero random variables and $r \geq 2$. Then, there exists a finite constant $c_{r}>0$ which is independent of $n$ such that

$$
\mathbb{E}\left[\left|\sum_{i=1}^{n} X_{i}\right|^{r}\right] \leq c_{r} n^{\frac{r}{2}-1} \mathbb{E}\left[\sum_{i=1}^{n}\left|X_{i}\right|^{r}\right]
$$

for all integers $n \geq 1$.

In order to apply the von Bahr-Esseen inequalities, random variables must be independent. We also need independence when we use Lyapunov's criterion to show the central limit theorem (Theorem 1.3). Thus, we introduce a localization argument that was introduced by Conus et al in [9]. This localization argument produces independent random variables which are close to the solution $u(t, x)$ of $(1.1)$.

Let us define intervals $I(x, t ; c):=[x-\sqrt{c t}, x+\sqrt{c t}]$ for some fixed constant $c>0$. Let $u^{(c, 0)}(t, x):=1$ for all $t \geq 0$ and $x \in \mathbb{R}$, and then define

$$
u^{(c, n+1)}(t, x)=1+\int_{(0, t) \times I(x, t: c)} p_{t-s}(y-x) \sigma\left(u^{(c, n)}(s, y)\right) W(d s d y),
$$

iteratively for all $n \geq 0$. It is easy to see that $u^{(c, n)}(t, x)$ and $u^{(c, n)}(t, y)$ have the same distribution and are independent whenever $|x-y| \geq 2 n \sqrt{c t}$. The following lemma which is basically Theorem 3.9 of [22] says that for appropriate $c$ and $n$, we can have independent random variables which approximate $u(t, x)$ (more precisely, see (3.23) and (3.24) in [22] with $\mu=1)$.

Lemma 2.3 (Localization of the solution). For all $t \geq 1$ and $k \geq 2$, there exists a constant $A>0$ such that $u^{(k)}(t, x):=u^{\left(A k^{2} t,\left\lceil A k^{2} t\right\rceil\right)}(t, x)$ satisfies the following: There exists a constant $C$ independent of $k, t$ such that

$$
\sup _{x \in \mathbb{R}} \mathbb{E}\left[\left|u(t, x)-u^{(k)}(t, x)\right|^{k}\right] \leq 2 C^{k} e^{-k^{3} t}
$$


Moreover, there exists a finite constant $c_{0}>0$ independent of $k$ and $t$ such that whenever nonrandom points $x_{1}, \ldots, x_{m} \in \mathbb{R}$ satisfy

$$
\min _{1 \leq i \neq j \leq m}\left|x_{i}-x_{j}\right|>c_{0} t^{2} k^{3}
$$

$u^{(k)}\left(t, x_{1}\right), \ldots, u^{(k)}\left(t, x_{m}\right)$ are i.i.d. random variables.

We note that (2.4) easily implies that for all $t \geq 1$ and $k \geq 2$

$$
e^{(\underline{\gamma}(k)+o(1)) t} \leq \mathbb{E}\left[\left|u^{(k)}(t, x)\right|^{k}\right] \leq e^{(\bar{\gamma}(k)+o(1)) t}
$$

as $t \rightarrow \infty$. In addition, by following exactly the same proof of Lemma 2.3, we can also see that $u^{(k)}\left(t, x_{1}\right)-u^{(k)}\left(s, x_{1}\right), \ldots, u^{(k)}\left(t, x_{m}\right)-u^{(k)}\left(s, x_{m}\right)$ are independent random variables whenever $\min _{1 \leq i \neq j \leq m}\left|x_{i}-x_{j}\right|>c_{0}(t \vee s)^{2} k^{3}$.

\subsection{Partitions of the interval $\Lambda_{L}:=[-L, L]$}

Let us introduce some partitions of the interval $\Lambda_{L}:=[-L, L]$, which was introduced by Ben Arous et al in [1], so that we can use the independence of $u^{(k)}(t, x)$ and $u^{(k)}(t, y)$ whenever $x$ and $y$ are far apart. Given $L>0$, let $0<L^{\prime}<L$ and $q:=\left\lfloor\frac{2 L}{L^{\prime}}\right\rfloor$. Define $L_{j}^{\prime}=L^{\prime}+\alpha_{j} 1_{\{1,2, \ldots, q\}}(j)$ for some $\alpha_{j} \in[0,1]$ so that $2 L=\sum_{j=1}^{q} L_{j}^{\prime}$. Note that $L^{\prime} \leq L_{j}^{\prime} \leq L^{\prime}+1$ for all $1 \leq j \leq q$. We now define a partition $\left\{\Lambda_{i}\right\}_{i=1}^{q}$ of $\Lambda_{L}$ as

$$
\Lambda_{i}=\left[-L+\sum_{j=1}^{i-1} L_{j}^{\prime},-L+\sum_{j=1}^{i} L_{j}^{\prime}\right] \text { for } i=1, \ldots, q,
$$

with the convention that $\sum_{j=1}^{0} L_{j}^{\prime}=0$. Let the index set $\mathcal{I}=\{1,2, \ldots, q\}$ and let us partition $\mathcal{I}$ into $\mathcal{I}_{1} \cup \mathcal{I}_{2}$, where $\mathcal{I}_{1}=\{i \in \mathcal{I}: i$ is even $\}$ and $\mathcal{I}_{2}=\{i \in \mathcal{I}: i$ is odd $\}$. Then, for $i, j \in \mathcal{I}_{\ell}$ with $i \neq j$, we have $d\left(\Lambda_{i}, \Lambda_{j}\right) \geq L^{\prime}$ for $\ell=1,2$. These partitions are heavily used in the proofs of Theorems 1.1 and 1.2 .

We now introduce another decomposition of $\Lambda_{L}$ for the proof of Theorem 1.3. Define subintervals $\Lambda_{i}^{\prime} \subset \Lambda_{i}$ as

$$
\Lambda_{i}^{\prime}=\left[-L+\sum_{j=1}^{i-1} L_{j}^{\prime}+\left\lceil c_{0} t^{2} k^{3}\right\rceil,-L+\sum_{j=1}^{i} L_{j}^{\prime}-\left\lceil c_{0} t^{2} k^{3}\right\rceil\right] \quad \text { for } i=1, \ldots, q
$$

and set the strip set $S_{L}:=\Lambda_{L} \backslash \bigcup_{i \in \mathcal{I}} \Lambda_{i}^{\prime}$ so that $\Lambda_{L}=S_{L} \cup \bigcup_{i \in \mathcal{I}} \Lambda_{i}^{\prime}$. We also partition $S_{L}$ as $\left\{S_{L, i}: i=0, \ldots, q\right\}$ where $S_{L, 0}:=\left[-L,-L+\left\lceil c_{0} t^{2} k^{3}\right\rceil\right], S_{L, q}:=\left[L-\left\lceil c_{0} t^{2} k^{3}\right\rceil, L\right]$ and

$$
S_{L, i}:=\left[-L+\sum_{j=1}^{i} L_{j}^{\prime}-\left\lceil c_{0} t^{2} k^{3}\right\rceil,-L+\sum_{j=1}^{i} L_{j}^{\prime}+\left\lceil c_{0} t^{2} k^{3}\right\rceil\right] \quad \text { for } i=1, \ldots, q-1 .
$$

Here, $c_{0}$ is the constant that appeared in Lemma 2.3. Note that $u^{(k)}(t, x)$ and $u^{(k)}(t, y)$ are independent as long as $x \in \Lambda_{i}^{\prime}$ and $y \in \Lambda_{j}^{\prime}$ or $x \in S_{L, i}$ and $y \in S_{L, j}$ for $i \neq j$.

For Theorems 1.1, 1.3 and 1.5, we basically take $L:=L(t):=e^{\lambda t}$ and $L^{\prime}:=L^{\prime}(t):=e^{\lambda^{\prime} t}$ for some constants $\lambda>\lambda^{\prime}>0$ (unless they are specified otherwise). On the other hand, we specify $L$ and $L^{\prime}$ for Theorem 1.2 in Section 4 . 


\section{Proof of Theorem 1.1}

In this section, we give a proof of Theorem 1.1 (the weak law of large numbers). We first show that the spatial average of $u^{(k)}$ over the interval $\Lambda_{L}:=[-L, L]$ is close to the spatial average of $u$ over the same interval $\Lambda_{L}$ for all large $t$, no matter what the interval length $\left(2 L:=\left|\Lambda_{L}\right|\right)$ can be.

Lemma 3.1. Let $\Lambda_{L}:=[-L, L]$. For every $\epsilon>0$ and $k \geq 2$, we have

$$
\lim _{t \rightarrow \infty} \sup _{L \geq 1} \mathbb{P}\left\{\left|\frac{1}{\left|\Lambda_{L}\right|} \int_{\Lambda_{L}}(u(t, x)-1) d x-\frac{1}{\left|\Lambda_{L}\right|} \int_{\Lambda_{L}}\left(u^{(k)}(t, x)-1\right) d x\right| \geq \epsilon\right\}=0
$$

Proof. Jensen's inequality and Lemma 2.3 implies that for all $t \geq 1$

$$
\begin{aligned}
\mathbb{E}\left[\left|\frac{1}{\left|\Lambda_{L}\right|} \int_{\Lambda_{L}}\left(u(t, x)-u^{(k)}(t, x)\right) d x\right|^{2}\right] & \leq \frac{1}{\left|\Lambda_{L}\right|} \int_{\Lambda_{L}} \mathbb{E}\left[\left|u(t, x)-u^{(k)}(t, x)\right|^{2}\right] d x \\
& \leq \sup _{x \in \mathbb{R}} \mathbb{E}\left[\left|u(t, x)-u^{(k)}(t, x)\right|^{2}\right] \\
& \leq \sup _{x \in \mathbb{R}} \mathbb{E}\left[\left|u(t, x)-u^{(k)}(t, x)\right|^{k}\right]^{\frac{2}{k}} \\
& \leq 2 C^{2} e^{-2 k^{2} t},
\end{aligned}
$$

where the constant $C$ is given in Lemma 2.3, which is independent of $L$. We now use Chebyshev's inequality to complete the proof.

The following lemma gives us an estimation of the moments of $\int_{\Lambda_{L}}\left(u^{(k)}(t, x)-1\right) d x$ in which Lemma 2.1 plays a crucial role. For simplicity, we consider in this section $\Lambda_{L}:=[-L, L]$, $L:=L(t):=e^{\lambda t}$ and $L^{\prime}:=e^{\lambda^{\prime} t}$ for some fixed constants $\lambda>\lambda^{\prime}>0$ (see also Section 2.2 for the partitions of $\Lambda_{L}$ ).

Lemma 3.2. For all $1 \leq r \leq 2$ and $k \geq 2$, we have

$$
\mathbb{E}\left[\left|\int_{\Lambda_{L}}\left(u^{(k)}(t, x)-1\right) d x\right|^{r}\right] \leq C_{r} \exp \left\{\left(\lambda+\lambda^{\prime}(r-1)+\bar{\gamma}(r)+o(1)\right) t\right\}
$$

as $t \rightarrow \infty$, where $C_{r}$ is a positive constant only depending $r$.

Proof. From Lemma 2.3, we know that $u^{(k)}(t, x)-1$ and $u^{(k)}(t, y)-1$ are independent whenever $x \in \Lambda_{i}$ and $y \in \Lambda_{j}$ with $i, j \in \mathcal{I}_{l}$ and $i \neq j$ for each $l=1,2$. Indeed, for all large enough $t$, $\left|\Lambda_{i}\right| \geq 2 e^{\lambda^{\prime} t} \gg c_{0} t^{2} k^{3}$ for all $k \geq 2$, which implies the independence. Once we decompose $\Lambda_{L}$ as

$$
\Lambda_{L}=\bigcup_{\ell=1,2} \bigcup_{i \in \mathcal{I}_{\ell}} \Lambda_{i}
$$

by using Jensen's inequality, Lemma 2.1 and Jensen's inequality, respectively, in lines two, three and four below, we see that 


$$
\begin{aligned}
\mathbb{E}\left[\left|\int_{\Lambda_{L}}\left(u^{(k)}(t, x)-1\right) d x\right|^{r}\right] & =\mathbb{E}\left[\left|\sum_{\ell=1,2} \sum_{i \in \mathcal{I}_{\ell}} \int_{\Lambda_{i}}\left(u^{(k)}(t, x)-1\right) d x\right|^{r}\right] \\
& \leq 2^{r-1} \sum_{\ell=1,2} \mathbb{E}\left[\left|\sum_{i \in \mathcal{I}_{\ell}} \int_{\Lambda_{i}}\left(u^{(k)}(t, x)-1\right) d x\right|^{r}\right] \\
& \leq 2^{r} \sum_{\ell=1,2} \sum_{i \in \mathcal{I}_{\ell}} \mathbb{E}\left[\left|\int_{\Lambda_{i}}\left(u^{(k)}(t, x)-1\right) d x\right|^{r}\right] \\
& \leq 2^{r} \sum_{\ell=1,2} \sum_{i \in \mathcal{I}_{\ell}}\left|\Lambda_{i}\right|^{r-1} \int_{\Lambda_{i}} \mathbb{E}\left[\left|u^{(k)}(t, x)-1\right|^{r}\right] d x .
\end{aligned}
$$

It is clear that from (2.6), for all $x \in \mathbb{R}$,

$$
\mathbb{E}\left[\left|u^{(k)}(t, x)-1\right|^{r}\right] \leq 2^{r} \mathbb{E}\left[\left|u^{(k)}(t, x)\right|^{r}\right] \leq 2^{r} e^{(\bar{\gamma}(r)+o(1)) t},
$$

as $t \rightarrow \infty$. Therefore, we conclude that

$$
\begin{aligned}
\mathbb{E}\left[\left|\int_{\Lambda_{L}}\left(u^{(k)}(t, x)-1\right) d x\right|^{r}\right] & \leq 2^{r} \sum_{k=1,2} \sum_{i \in \mathcal{I}_{k}}\left|\Lambda_{i}\right|^{r-1} \int_{\Lambda_{i}} 2^{r} \mathbb{E}\left[\left|u^{(k)}(t, x)\right|^{r}\right] d x \\
& \leq C_{r}\left(L^{\prime}+1\right)^{r-1}(2 L) e^{(\bar{\gamma}(r)+o(1)) t}
\end{aligned}
$$

since $\left|\Lambda_{i}\right| \leq L^{\prime}+1$ for all $i$.

We are now ready to prove Theorem 1.1.

Proof of Theorem 1.1. We first note that thanks to Lemma 3.1, it suffices to show that the weak law of large numbers (i.e. (1.6)) holds for $u^{(k)}(t, x)$. Since $\left|\Lambda_{L}\right|=2 L(t)=2 e^{\lambda t}$, Lemma 3.2 implies that, for $0<\delta<1$, as $t \rightarrow \infty$, we have

$$
\mathbb{E}\left[\left|\frac{1}{\left|\Lambda_{L}\right|} \int_{\Lambda_{L}}\left(u^{(k)}(t, x)-1\right) d x\right|^{1+\delta}\right] \leq C_{\delta} \exp \left\{\delta\left(\lambda^{\prime}-\lambda+\frac{\bar{\gamma}(1+\delta)}{\delta}+o(1)\right) t\right\},
$$

where $C_{\delta}$ is a positive constant only depending on $\delta$. Since $\lambda>\bar{\gamma}^{\prime}(1)$ and $\bar{\gamma}(1)=0$, we can take $\lambda^{\prime}$ and $\delta$ sufficiently small so that

$$
\lambda^{\prime}-\lambda+\frac{\bar{\gamma}(1+\delta)-\bar{\gamma}(1)}{\delta}<-c .
$$

for some positive constant $c>0$. Then, by Chebyshev's inequality, taking $t \rightarrow \infty$ leads to the desired conclusion.

\section{Proof of Theorem 1.2}

In this section, we provide a proof of Theorem 1.2 (the strong law of large numbers). The following proposition tells us the continuity of the solution to (1.1), which helps us control the fluctuation of $\int_{\Lambda_{L}}(u(t, x)-1) d x$ in time. In this section, we write $L(t)$ and $L(s)$ instead of $L$ where $L(t)=e^{\lambda t}$ and $L(s)=e^{\lambda s}$. 
Lemma 4.1. There exists a finite positive constant $D$ which is independent of $n$ such that for all $k \geq 2$

$$
\sup _{n \leq t \neq s<n+1} \sup _{x \in \mathbb{R}} \mathbb{E}\left[|u(t, x)-u(s, x)|^{k}\right] \leq D^{k} e^{(\bar{\gamma}(k)+o(1)) n}|t-s|^{\frac{k}{4}},
$$

as $n \rightarrow \infty$.

The proof is very similar to that of Lemma 5.4 of [20]. To the best of our knowledge, however, the exact coefficient $e^{\bar{\gamma}(k) n}$ has not been exactly identified. Thus we briefly present the proof of Lemma 4.1 for the sake of completeness.

Proof. From the proof of Lemma 5.4 in [20] with $\Phi_{t}(x)=\sigma(u(t, x))$, for all $x \in \mathbb{R}$ and $0<s<t$, we have

$$
\mathbb{E}\left[|u(t, x)-u(s, x)|^{k}\right]^{\frac{2}{k}} \leq 2\left(J_{1}+J_{2}\right),
$$

where

$$
\begin{aligned}
& J_{1}:=\text { const } \cdot k \int_{0}^{t} \int_{\mathbb{R}}\left[p_{t-r}(y-x)-p_{s-r}(y-x)\right]^{2} \mathbb{E}\left[\sigma^{k}(u(r, y))\right]^{\frac{2}{k}} d y d s, \\
& J_{2}:=\text { const } \cdot k \int_{s}^{t} \int_{\mathbb{R}}\left[p_{t-r}(y-x)\right]^{2} \mathbb{E}\left[\sigma^{k}(u(r, y))\right]^{\frac{2}{k}} d y d s .
\end{aligned}
$$

Here, (1.3) implies that as $t \rightarrow \infty$,

$$
\sup _{0 \leq r \leq t} \sup _{y \in \mathbb{R}} \mathbb{E}\left[\sigma^{k}(u(r, y))\right] \leq e^{(\bar{\gamma}(k)+o(1)) t} .
$$

In addition, we can also have that (see [20, Section 3.3.1])

$$
\int_{0}^{t} \int_{\mathbb{R}}\left[p_{t-r}(y-x)-p_{s-r}(y-x)\right]^{2} d y d s+\int_{s}^{t} \int_{\mathbb{R}}\left[p_{t-r}(y-x)\right]^{2} d y d s \leq C|t-s|^{\frac{1}{2}},
$$

where the constant $C$ is independent of $t, s$ and $x$. Putting all the things together, we get (4.1).

We set $L(t):=e^{\lambda t}, L^{\prime}(t):=e^{\lambda^{\prime} t}$ and $\Lambda_{L(t)}:=[-L(t), L(t)]$ for fixed constants $\lambda>\lambda^{\prime}>0$. We now control the fluctuation of $\int_{\Lambda_{L(t)}}(u(t, x)-1) d x$ in time. For simplicity, we define

$$
X(t):=\int_{\Lambda_{L(t)}}(u(t, x)-1) d x
$$

Lemma 4.2. For any $p>4$, there exists a constant $C>0$ which only depends on $p$ and $\lambda$ such that as $n \rightarrow \infty$

$$
\mathbb{E}\left[\sup _{t \neq s \in[n, n+1)}|X(t)-X(s)|^{p}\right] \leq C \exp \left\{\left(\frac{\lambda p}{2}+\frac{\lambda^{\prime} p}{2}+\bar{\gamma}(p)+o(1)\right) n\right\},
$$

provided that

$$
\frac{2 \lambda}{5}<\lambda^{\prime}<\lambda
$$


Proof. By a quantitative form of the Kolmogorov continuity theorem (see e.g. [20, Theorem C.6]), it is enough to show that for some $p_{2} \in\left(1, \frac{p}{4}\right)$, there exists a constant $C_{\lambda, p}$ only depending on $\lambda$ and $p$ such that

$$
\sup _{t \neq s \in[n, n+1)} \mathbb{E}\left[\frac{|X(t)-X(s)|^{p}}{|t-s|^{p_{2}}}\right] \leq C_{\lambda, p} \exp \left\{\left(\frac{\lambda p}{2}+\frac{\lambda^{\prime} p}{2}+\bar{\gamma}(p)+o(1)\right) n\right\},
$$

as $n \rightarrow \infty$. We now fix large $n$ and $t \neq s \in[n, n+1)$. Without loss of generality, we assume that $t>s$. We estimate the LHS of (4.5) as follows:

$$
\mathbb{E}\left[|X(t)-X(s)|^{p}\right] \leq 4^{p}\left(A_{1}+A_{2}+A_{3}+A_{4}\right),
$$

where

$$
\begin{aligned}
& A_{1}:=\mathbb{E}\left[\left|\int_{\Lambda_{L(t)}}\left(u(t, x)-u^{(k)}(t, x)\right) d x\right|^{p}\right], \\
& A_{2}:=\mathbb{E}\left[\left|\int_{\Lambda_{L(s)}}\left(u(s, x)-u^{(k)}(s, x)\right) d x\right|^{p}\right], \\
& A_{3}:=\mathbb{E}\left[\left|\int_{\Lambda_{L(s)}}\left(u^{(k)}(t, x)-u^{(k)}(s, x)\right) d x\right|^{p}\right], \\
& A_{4}:=\mathbb{E}\left[\left|\int_{\Lambda_{L(t)} \backslash \Lambda_{L(s)}}\left(u^{(k)}(t, x)-1\right) d x\right|^{p}\right],
\end{aligned}
$$

where $k$ will be specified later (see (4.7) below). We will denote by $C$ a constant depending only on $p$ and $\lambda$ and can be changed line by line. By Jensen's inequality and Hölder's inequality, we have

$$
A_{1} \leq C e^{\lambda p t} \sup _{x \in \mathbb{R}} \mathbb{E}\left[\left|u(t, x)-u^{(k)}(t, x)\right|^{p}\right] \leq C e^{\lambda p t} \sup _{x \in \mathbb{R}} \mathbb{E}\left[\left|u(t, x)-u^{(k)}(t, x)\right|^{k}\right]^{\frac{p}{k}},
$$

for all $k \geq p$. If we choose $k$ as

$$
k:=k(n,|t-s|):=p \vee\left(\frac{\lambda-\lambda^{\prime}}{2}-\frac{\bar{\gamma}(p)}{p}-\frac{p_{2}}{n p} \ln |t-s|\right)^{\frac{1}{2}}
$$

then Lemma 2.3 implies that for all $t \neq s \in[n, n+1)$,

$$
\sup _{x \in \mathbb{R}} \mathbb{E}\left[\left|u(t, x)-u^{(k)}(t, x)\right|^{p}\right] \leq C|t-s|^{p_{2}} e^{\left(\bar{\gamma}(p)-\frac{\lambda p}{2}+\frac{\lambda^{\prime} p}{2}\right) n},
$$

which in turn implies that

$$
A_{1} \leq C|t-s|^{p_{2}} \exp \left\{\left(\frac{\lambda p}{2}+\frac{\lambda^{\prime} p}{2}+\bar{\gamma}(p)\right) n\right\} .
$$

Similarly, we can estimate $A_{2}$ so that we have

$$
A_{2} \leq C|t-s|^{p_{2}} \exp \left\{\left(\frac{\lambda p}{2}+\frac{\lambda^{\prime} p}{2}+\bar{\gamma}(p)\right) n\right\} .
$$


Now we estimate $A_{3}$. Let $\beta$ be a fixed positive constant that will be specified later. We first consider the case where $t \neq s \in[n, n+1)$ with $|t-s| \geq e^{-\beta n}$. In this case, it is easy to see that for all large $n$

$$
e^{\lambda^{\prime} n} \gg c_{0} k^{3} t^{2}
$$

where $c_{0}$ was the constant that appeared in Lemma 2.3 and $k:=k(n,|t-s|)$ was defined in (4.7). Therefore, we can use the generalized von Bahr-Esseen inequality (Lemma 2.2) as in the proof of Lemma 3.2. With the same notations (with $s$ instead of $t$ ) as in the proof of Lemma 3.2 , we get that

$$
\begin{aligned}
A_{3} & =\mathbb{E}\left[\left|\sum_{\ell=1,2} \sum_{i \in \mathcal{I}_{\ell}} \int_{\Lambda_{i}}\left(u^{(k)}(t, x)-u^{(k)}(s, x)\right) d x\right|^{p}\right] \\
& \leq 2^{p-1} \sum_{\ell=1,2} \mathbb{E}\left[\left|\sum_{i \in \mathcal{I}_{\ell}} \int_{\Lambda_{i}}\left(u^{(k)}(t, x)-u^{(k)}(s, x)\right) d x\right|^{p}\right] \\
& \leq C\left(\frac{L(s)}{L^{\prime}(s)}\right)^{\frac{p}{2}-1} \sum_{\ell=1,2} \sum_{i \in \mathcal{I}_{\ell}} \mathbb{E}\left[\left|\int_{\Lambda_{i}}\left(u^{(k)}(t, x)-u^{(k)}(s, x)\right) d x\right|^{p}\right] \\
& \leq C\left(\frac{L(s)}{L^{\prime}(s)}\right)^{\frac{p}{2}-1} \sum_{\ell=1,2} \sum_{i \in \mathcal{I}_{\ell}}\left(L^{\prime}(s)\right)^{p-1} \int_{\Lambda_{i}} \mathbb{E}\left[\left|u^{(k)}(t, x)-u^{(k)}(s, x)\right|^{p}\right] d x \\
& \leq C e^{\left(\frac{\lambda p}{2}+\frac{\lambda^{\prime} p}{2}\right) n} \sup _{x \in \mathbb{R}} \mathbb{E}\left[\left|u^{(k)}(t, x)-u^{(k)}(s, x)\right|^{p}\right] .
\end{aligned}
$$

Note that for all $x \in \mathbb{R}$,

$$
\begin{aligned}
& \mathbb{E}\left[\left|u^{(k)}(t, x)-u^{(k)}(s, x)\right|^{p}\right] \\
& \quad \leq C\left\{\mathbb{E}\left[\left|u^{(k)}(t, x)-u(t, x)\right|^{p}\right]+\mathbb{E}\left[\left|u^{(k)}(s, x)-u(s, x)\right|^{p}\right]+\mathbb{E}\left[|u(t, x)-u(s, x)|^{p}\right]\right\} .
\end{aligned}
$$

Here, the first two terms on the right hand side above can be bounded as in (4.8). On the other hand, Lemma 4.1 implies that the last term is bounded by $D^{p} e^{(\bar{\gamma}(p)+o(1)) n}|t-s|^{\frac{p}{4}}$. Since $p_{2}<p / 4$, as long as $|t-s| \geq e^{-\beta n}$, we get

$$
A_{3} \leq|t-s|^{p_{2}} \exp \left\{\left(\frac{\lambda p}{2}+\frac{\lambda^{\prime} p}{2}+\bar{\gamma}(p)+o(1)\right) n\right\} .
$$

We now consider $A_{3}$ when $0<|t-s|<e^{-\beta n}$. First of all, by Jensen's inequality,

$$
A_{3} \leq C e^{\lambda p n} \sup _{x \in \mathbb{R}} \mathbb{E}\left[\left|u^{(k)}(t, x)-u^{(k)}(s, x)\right|^{p}\right] .
$$

As in (4.11), (4.8) makes us only bound the term $e^{\lambda p n} \sup _{x \in \mathbb{R}} \mathbb{E}\left[|u(t, x)-u(s, x)|^{p}\right]$. Once again, by Lemma 4.1, we have

$$
\sup _{x \in \mathbb{R}} \mathbb{E}\left[|u(t, x)-u(s, x)|^{p}\right] \leq D^{p} e^{(\bar{\gamma}(p)+o(1)) n}|t-s|^{\frac{p}{4}} .
$$


Since $|t-s|<e^{-\beta n}$, we choose $\beta>2\left(\lambda-\lambda^{\prime}\right) p /\left(p-4 p_{2}\right)$ to get that

$$
D^{p} e^{(\bar{\gamma}(p)+\lambda p) n}|t-s|^{\frac{p}{4}} \leq e^{\left(\frac{\lambda p}{2}+\frac{\lambda^{\prime} p}{2}+\bar{\gamma}(p)\right) n}|t-s|^{p_{2}},
$$

and this implies (4.13) when $|t-s|<e^{-\beta n}$.

Finally, we estimate $A_{4}$. Note that for $x \in[0,1]$, there exist constants $\lambda_{1}$ and $\lambda_{2}$ which only depend on $\lambda$ such that $\lambda_{1} x \leq 1-e^{-\lambda x} \leq \lambda_{2} x$. Thus, we have

$$
\lambda_{1}(t-s) e^{\lambda t} \leq L(t)-L(s) \leq \lambda_{2}(t-s) e^{\lambda t} .
$$

Let $\eta$ be a fixed positive constant that will be specified later. For $0<|t-s| \leq e^{-\eta n}$, by Jensen's inequality, we have

$$
\begin{aligned}
A_{4} & \leq\left(e^{\lambda t} \lambda_{2}(t-s)\right)^{p} \sup _{x \in \mathbb{R}} \mathbb{E}\left[\left|u^{(k)}(t, x)-1\right|^{p}\right] \\
& \leq C e^{\lambda p n}|t-s|^{p} e^{(\bar{\gamma}(p)+o(1)) n} \\
& \leq \exp \left\{\left(\frac{\lambda p}{2}+\frac{\lambda^{\prime} p}{2}+\bar{\gamma}(p)+o(1)\right) n\right\}|t-s|^{p_{2}},
\end{aligned}
$$

as $n \rightarrow \infty$, provided that

$$
\frac{\lambda p}{2}-\frac{\lambda^{\prime} p}{2} \leq \eta\left(p-p_{2}\right)
$$

We now consider $A_{4}$ when $e^{-\eta n} \leq t-s \leq 1$. As we bound $A_{3}$ when $|t-s|$ is not too small, we again use the generalized von Bahr-Esseen inequality (Lemma 2.2). Here, we divide the interval of length $L(t)-L(s)$ into the subintervals of length $e^{\lambda^{\prime} n}(t-s)$. We also assume $\eta<\lambda^{\prime}$ so that for all large $n$

$$
e^{\lambda^{\prime} n}(t-s) \gg c_{0} t^{2} k^{3}
$$

where $k$ is in (4.7). This guarantees the independence between $u^{(k)}(t, x)$ and $u^{(k)}(t, y)$ when $x \in \Lambda_{i}$ and $y \in \Lambda_{j}$ with $i, j \in \mathcal{I}_{l}$ and $i \neq j$ for each $l=1,2$. As for $A_{3}$ (see (4.10), by considering $L$ and $L^{\prime}$ as $e^{\lambda t}-e^{\lambda s}$ and $e^{\lambda^{\prime} t}(t-s)$, we have

$$
\begin{aligned}
A_{4} & \leq C e^{\left(\frac{\lambda p}{2}+\frac{\lambda^{\prime} p}{2}\right) n}|t-s|^{p} \sup _{x \in \mathbb{R}} \mathbb{E}\left[\left|u^{(k)}(t, x)-1\right|^{p}\right] \\
& \leq C|t-s|^{p} \exp \left\{\left(\frac{\lambda p}{2}+\frac{\lambda^{\prime} p}{2}+\bar{\gamma}(p)+o(1)\right) n\right\} \\
& \leq C|t-s|^{p_{2}} \exp \left\{\left(\frac{\lambda p}{2}+\frac{\lambda^{\prime} p}{2}+\bar{\gamma}(p)+o(1)\right) n\right\} .
\end{aligned}
$$

Therefore, the only remaining thing is to verify (4.16) for some $\eta \in\left(0, \lambda^{\prime}\right)$ and $p_{2} \in(1, p / 4)$. Using the assumption that $\lambda^{\prime} \in(2 \lambda / 5, \lambda)$, this can be easily done by choosing $p_{2}=p / 4-\epsilon_{1}$ and $\eta=\lambda^{\prime}-\epsilon_{2}$ for some small $\epsilon_{1}, \epsilon_{2}>0$.

We now provide the proof of Theorem 1.2. 
Proof of Theorem 1.2. We first consider $\mathbb{E}|X(n)|^{p}$ for $p>4$ where $X(t):=\int_{\Lambda_{L(t)}}(u(t, x)-1) d x$. Similar to Lemma 3.2, we use Jensen's inequality, the generalized von Bahr-Esseen inequality (Lemma 2.2) and Lemma 2.3 to get that, as $n \rightarrow \infty$,

$$
\begin{aligned}
\mathbb{E}\left[|X(n)|^{p}\right] & =\mathbb{E}\left[\left|\int_{\Lambda_{L(n)}}(u(n, x)-1) d x\right|^{p}\right] \\
& \leq C\left\{\mathbb{E}\left[\left|\int_{\Lambda_{L(n)}}\left(u(n, x)-u^{(k)}(n, x)\right) d x\right|^{p}\right]+\mathbb{E}\left[\left|\int_{\Lambda_{L(n)}}\left(u^{(k)}(n, x)-1\right) d x\right|^{p}\right]\right\} \\
& \leq C\left\{e^{\lambda p n} \sup _{x \in \mathbb{R}} \mathbb{E}\left[\left|u(n, x)-u^{(k)}(n, x)\right|^{p}\right]+e^{\left(\frac{\lambda p}{2}+\frac{\lambda^{\prime} p}{2}\right) n} \sup _{x \in \mathbb{R}} \mathbb{E}\left[\left|u^{(k)}(n, x)-1\right|^{p}\right]\right\} \\
& \leq C\left\{\exp \left(\left(\lambda p-k^{2} p\right) n\right)+\exp \left(\left(\frac{\lambda p}{2}+\frac{\lambda^{\prime} p}{2}+\bar{\gamma}(p)+o(1)\right) n\right)\right\} \\
& \leq C \exp \left(\left(\frac{\lambda p}{2}+\frac{\lambda^{\prime} p}{2}+\bar{\gamma}(p)+o(1)\right) n\right),
\end{aligned}
$$

where $C>0$ is a constant only depending $p$ and $\lambda$, which can be changed line by line. Of course, we take $k>p \vee \sqrt{\lambda}$ in the last inequality above. Combining this with Lemma 4.2, we have

$$
\begin{aligned}
\mathbb{E}\left[\sup _{t \in[n, n+1)}|X(t)|^{p}\right] & \leq 2^{p}\left\{\mathbb{E}\left[|X(n)|^{p}\right]+\mathbb{E}\left[\sup _{t \neq s \in[n, n+1]}|X(t)-X(s)|^{p}\right]\right\} \\
& \leq C \exp \left(\left(\frac{\lambda p}{2}+\frac{\lambda^{\prime} p}{2}+\bar{\gamma}(p)+o(1)\right) n\right),
\end{aligned}
$$

where $\lambda^{\prime} \in\left(\frac{2 \lambda}{5}, \lambda\right)$ and $p>4$. Hence, for any $\epsilon>0$, we have that as $n \rightarrow \infty$

$$
\begin{aligned}
& \mathbb{P}\left\{\sup _{t \in[n, n+1]} \frac{1}{\left|\Lambda_{L(t)}\right|} \int_{\Lambda_{L(t)}}(u(t, x)-1) d x>\epsilon\right\} \\
& \leq \frac{\mathbb{E}\left[\sup _{t \in[n, n+1]}|X(t)|^{p}\right]}{\left|\Lambda_{L(n)}\right|^{p} \epsilon^{p}} \\
& \leq C \epsilon^{-p} \exp \left(\left(-\frac{\lambda p}{2}+\frac{\lambda^{\prime} p}{2}+\bar{\gamma}(p)+o(1)\right) n\right) .
\end{aligned}
$$

As long as $\lambda>\frac{5 \bar{\gamma}(4)}{6}$, we can choose $\lambda^{\prime}$ close to $\frac{2 \lambda}{5}$ and $p$ close to 4 so that $-\frac{\lambda p}{2}+\frac{\lambda^{\prime} p}{2}+\bar{\gamma}(p)<0$. In other words, if $\lambda>\frac{5 \bar{\gamma}(4)}{6}$, (4.17) is summable over $n$, and the Borel-Cantelli lemma completes the proof.

\section{Proof of Theorem 1.3}

In this section, we provide a proof of Theorem 1.3 by following the strategy for the proof of the central limit theorem given in Cranston-Molchanov [10]. We first estimate the variance of $\int_{\Lambda_{L}} u(t, x) d x$ as $t \rightarrow \infty$ where $\Lambda_{L}:=[-L, L]$ and $L:=L(t)$. For the estimation of the variance 
of $\int_{-R}^{R} u(t, x) d x$ for a fixed time $t>0$ but as $R \rightarrow \infty$ is given in Proposition 3.1 in [18]. From now on, $L$ always denote $L(t)$.

Lemma 5.1. Suppose $L(t)>0$ is an increasing function such that

$$
\liminf _{t \rightarrow \infty} \frac{L(t)}{\sqrt{t}}=\infty
$$

Then we have

$$
\lim _{t \rightarrow \infty} \frac{\operatorname{Var}\left(\int_{\Lambda_{L}} u(t, x) d x\right)}{2 L(t) \int_{0}^{t} \xi(r) d r}=1
$$

where $\xi(r):=\mathbb{E}\left[\sigma^{2}(u(r, 0))\right]$.

Proof. The proof is similar to Proposition 3.1 in [18]. Due to the Itô isometry, we have

$$
\begin{aligned}
\mathbb{E}\left[u(t, x) u\left(t, x^{\prime}\right)\right] & =1+\int_{0}^{t} \int_{\mathbb{R}} p_{t-r}(x-y) p_{t-r}\left(x^{\prime}-y\right) \mathbb{E}\left[\sigma^{2}(u(r, y))\right] d y d r \\
& =1+\int_{0}^{t} \int_{\mathbb{R}} \xi(r) p_{t-r}(x-y) p_{t-r}\left(x^{\prime}-y\right) d y d r \\
& =1+\int_{0}^{t} \xi(r) p_{2 t-2 r}\left(x-x^{\prime}\right) d r
\end{aligned}
$$

where we have used the semigroup property in the last equality. Therefore, we have

$$
\begin{aligned}
\operatorname{Var}\left(\int_{\Lambda_{L}} u(t, x) d x\right) & =\int_{-L(t)}^{L(t)} \int_{-L(t)}^{L(t)} \int_{0}^{t} \xi(r) p_{2 t-2 r}\left(x-x^{\prime}\right) d r d x d x^{\prime} \\
& =2 \int_{0}^{t} \xi(r) \int_{0}^{2 L(t)} p_{2 t-2 r}(z)(2 L(t)-z) d z d r \\
& =2 L(t) \int_{0}^{t} \xi(r) \int_{0}^{2 L(t)} p_{2 t-2 r}(z)\left(2-\frac{z}{L(t)}\right) d z d r .
\end{aligned}
$$

First of all, it is easy to see that

$$
\sup _{0 \leq r \leq t} \frac{1}{L(t)}\left|\int_{0}^{2 L(t)} z p_{2 t-2 r}(z) d z\right| \leq \frac{\sqrt{t}}{L(t)} \rightarrow 0 \quad \text { as } t \rightarrow \infty .
$$

In addition, we also have

$$
2 \int_{0}^{2 L(t)} p_{2 t-2 r}(z) d z=1-2 \int_{2 L(t)}^{\infty} p_{2 t-2 r}(z) d z
$$

and

$$
\sup _{0 \leq r \leq t}\left|\int_{2 L(t)}^{\infty} p_{2 t-2 r}(z) d z\right| \leq \exp \left(-L^{2}(t) / t\right) \rightarrow 0 \quad \text { as } t \rightarrow \infty .
$$

Combining things together, we get the desired result. 
The next lemma shows that it is enough to prove the central limit theorem for the timedependent average of the localized random field $\left\{u^{(k)}(t, x)\right\}$ which has independent property as long as $x$ and $y$ are far apart (see Lemma 2.3).

Lemma 5.2. Let $L=L(t)=e^{\lambda t}$ for any $\lambda>0$. For all $\epsilon>0$ and sufficiently large $k$, we have

$$
\lim _{t \rightarrow \infty} \mathbb{P}\left\{\left|\frac{\int_{\Lambda_{L}}(u(t, x)-1) d x}{\sqrt{\operatorname{Var}\left(\int_{\Lambda_{L}} u(t, x) d x\right)}}-\frac{\int_{\Lambda_{L}}\left(u^{(k)}(t, x)-1\right) d x}{\sqrt{\operatorname{Var}\left(\int_{\Lambda_{L}} u^{(k)}(t, x) d x\right)}}\right| \geq \epsilon\right\}=0 .
$$

Proof. We first show the following: For $k>(\sqrt{\bar{\gamma}(2) / 2+2 \lambda} \vee 2)$ and all large $t>0$,

$$
\left|\operatorname{Var}\left(\int_{\Lambda_{L}} u(t, x) d x\right)-\operatorname{Var}\left(\int_{\Lambda_{L}} u^{(k)}(t, x) d x\right)\right| \leq e^{-c(k) t},
$$

where $c(k)>0$ is an increasing function of $k$ such that $\lim _{k \rightarrow \infty} c(k)=\infty$. To get this, we compute

$$
\begin{aligned}
& \left|\operatorname{Var}\left(\int_{\Lambda_{L}} u(t, x) d x\right)-\operatorname{Var}\left(\int_{\Lambda_{L}} u^{(k)}(t, x) d x\right)\right| \\
& =\left|\mathbb{E}\left[\left(\int_{\Lambda_{L}}(u(t, x)-1) d x\right)^{2}-\left(\int_{\Lambda_{L}}\left(u^{(k)}(t, x)-1\right) d x\right)^{2}\right]\right| \\
& =\left|\mathbb{E}\left[\left(\int_{\Lambda_{L}}\left(u(t, x)-u^{(k)}(t, x)\right) d x\right)\left(\int_{\Lambda_{L}}\left(u(t, x)+u^{(k)}(t, x)-2\right) d x\right)\right]\right| \\
& \leq \mathbb{E}\left[\left(\int_{\Lambda_{L}}\left(u(t, x)-u^{(k)}(t, x)\right) d x\right)^{2}\right]^{\frac{1}{2}} \mathbb{E}\left[\left(\int_{\Lambda_{L}}\left(u(t, x)+u^{(k)}(t, x)-2\right) d x\right)^{2}\right]^{\frac{1}{2}} \\
& \leq\left|\Lambda_{L}\right|^{2} \sup _{x \in \mathbb{R}} \mathbb{E}\left[\left|u(t, x)-u^{(k)}(t, x)\right|^{2}\right]^{\frac{1}{2}} \sup _{x \in \mathbb{R}} \mathbb{E}\left[\left|u(t, x)+u^{(k)}(t, x)-2\right|^{2}\right]^{\frac{1}{2}} \\
& \leq C \exp \left\{\left(2 \lambda+\frac{\bar{\gamma}(2)}{2}-k^{2}+o(1)\right) t\right\},
\end{aligned}
$$

as $t \rightarrow \infty$. Here, we used the Cauchy-Schwarz inequality, Jensen's inequality and Lemma 2.3 to get the last inequality above. This proves (5.3).

Now we define two terms

$$
\begin{aligned}
& A_{1}:=\left|\frac{\int_{\Lambda_{L}}(u(t, x)-1) d x}{\sqrt{\operatorname{Var}\left(\int_{\Lambda_{L}} u(t, x) d x\right)}}-\frac{\int_{\Lambda_{L}}\left(u^{(k)}(t, x)-1\right) d x}{\sqrt{\operatorname{Var}\left(\int_{\Lambda_{L}} u(t, x) d x\right)}}\right|, \\
& A_{2}:=\left|\frac{\int_{\Lambda_{L}}\left(u^{(k)}(t, x)-1\right) d x}{\sqrt{\operatorname{Var}\left(\int_{\Lambda_{L}} u(t, x) d x\right)}}-\frac{\int_{\Lambda_{L}}\left(u^{(k)}(t, x)-1\right) d x}{\sqrt{\operatorname{Var}\left(\int_{\Lambda_{L}} u^{(k)}(t, x) d x\right)}}\right| .
\end{aligned}
$$


To show (5.2), it is enough to show $A_{1}, A_{2} \rightarrow 0$ in probability as $t \rightarrow \infty$. For $A_{1}$, we have

$$
\begin{aligned}
\mathbb{E}\left[A_{1}\right] & \leq \frac{\left|\Lambda_{L}\right| \sup _{x \in \mathbb{R}} \mathbb{E}\left[\left|u(t, x)-u^{(k)}(t, x)\right|\right]}{\sqrt{\operatorname{Var}\left(\int_{\Lambda_{L}} u(t, x) d x\right)}} \\
& \leq \frac{e^{\lambda t} \sup _{x \in \mathbb{R}} \mathbb{E}\left[\left|u(t, x)-u^{(k)}(t, x)\right|^{k}\right]^{1 / k}}{\sqrt{\operatorname{Var}\left(\int_{\Lambda_{L}} u(t, x) d x\right)}} .
\end{aligned}
$$

Thus, Lemmas 2.3 and 5.1 implies that $\mathbb{E}\left[A_{1}\right] \rightarrow 0$ as $t \rightarrow \infty$.

We now estimate $\mathbb{E}\left[A_{2}\right]$. By Jensen's inequality, we obtain that

$$
\begin{aligned}
\mathbb{E}\left[A_{2}\right] & \leq \mathbb{E}\left[\left|\int_{\Lambda_{L}}\left(u^{(k)}(t, x)-1\right) d x\right|\right] \cdot\left|\frac{1}{\sqrt{\operatorname{Var}\left(\int_{\Lambda_{L}} u(t, x) d x\right)}}-\frac{1}{\sqrt{\operatorname{Var}\left(\int_{\Lambda_{L}} u^{(k)}(t, x) d x\right)}}\right| \\
& \leq \sqrt{\operatorname{Var}\left(\int_{\Lambda_{L}} u^{(k)}(t, x) d x\right)} \cdot\left|\frac{1}{\sqrt{\operatorname{Var}\left(\int_{\Lambda_{L}} u(t, x) d x\right)}}-\frac{1}{\sqrt{\operatorname{Var}\left(\int_{\Lambda_{L}} u^{(k)}(t, x) d x\right)}}\right| .
\end{aligned}
$$

Moreover,

$$
\begin{aligned}
& \left|\frac{1}{\sqrt{\operatorname{Var}\left(\int_{\Lambda_{L}} u(t, x) d x\right)}}-\frac{1}{\sqrt{\operatorname{Var}\left(\int_{\Lambda_{L}} u^{(k)}(t, x) d x\right)}}\right| \\
& =\frac{\left|\operatorname{Var}\left(\int_{\Lambda_{L}} u^{(k)}(t, x) d x\right)-\operatorname{Var}\left(\int_{\Lambda_{L}} u(t, x) d x\right)\right|}{\sqrt{\operatorname{Var}\left(\int_{\Lambda_{L}} u^{(k)}(t, x) d x\right) \operatorname{Var}\left(\int_{\Lambda_{L}} u(t, x) d x\right)}\left(\sqrt{\operatorname{Var}\left(\int_{\Lambda_{L}} u^{(k)}(t, x) d x\right)}+\sqrt{\operatorname{Var}\left(\int_{\Lambda_{L}} u(t, x) d x\right)}\right)} .
\end{aligned}
$$

Therefore, we can deduce $\mathbb{E}\left[A_{2}\right] \rightarrow 0$ as $t \rightarrow \infty$ by considering (5.3) and Lemma 5.1.

We now prove Theorem 1.3. For simplicity, we write $\Lambda_{L}:=[-L, L], L:=e^{\lambda t}$ and $L^{\prime}:=e^{\lambda^{\prime} t}$.

Proof of Theorem 1.3. We first show (i) of Theorem 1.3. By Lemma 5.2, it suffices to show that for sufficiently large $k$,

$$
\frac{\int_{\Lambda_{L}}\left(u^{(k)}(t, x)-1\right) d x}{\sqrt{\operatorname{Var}\left(\int_{\Lambda_{L}} u^{(k)}(t, x) d x\right)}} \stackrel{\mathcal{L}}{\rightarrow} N(0,1), \quad \text { as } t \rightarrow \infty .
$$

Using the decomposition of $\Lambda_{L}=S_{L} \cup \bigcup_{i \in \mathcal{I}} \Lambda_{i}^{\prime}$ (see (2.8)), we have

$$
\frac{\int_{\Lambda_{L}}\left(u^{(k)}(t, x)-1\right) d x}{\sqrt{\operatorname{Var}\left(\int_{\Lambda_{L}} u^{(k)}(t, x) d x\right)}}=\frac{\int_{S_{L}}\left(u^{(k)}(t, x)-1\right) d x}{\sqrt{\operatorname{Var}\left(\int_{\Lambda_{L}} u^{(k)}(t, x) d x\right)}}+\frac{\sum_{i \in \mathcal{I}} \int_{\Lambda_{i}^{\prime}}\left(u^{(k)}(t, x)-1\right) d x}{\sqrt{\operatorname{Var}\left(\int_{\Lambda_{L}} u^{(k)}(t, x) d x\right)}} .
$$


We claim that the first term on the RHS of (5.6) converges to 0 in probability. To show this, we use a partition $\left\{S_{L, i}: i=0, \ldots, q:=\left\lfloor\frac{2 L}{L^{\prime}}\right\rfloor\right\}$ of $S_{L}$ (see $\left.(2.9)\right)$. Since $\left\{\int_{S_{L, i}}\left(u^{(k)}(t, x)-1\right) d x\right\}_{i=0}^{q}$ is a collection of independent random variables by Lemma 2.3, we have

$$
\begin{aligned}
\mathbb{E}\left[\left|\int_{S_{L}}\left(u^{(k)}(t, x)-1\right) d x\right|^{2}\right] & =\sum_{i=0}^{q} \mathbb{E}\left[\left|\int_{S_{L, i}}\left(u^{(k)}(t, x)-1\right) d x\right|^{2}\right] \\
& \leq 4(q+1) \mathbb{E}\left[\left|\int_{0}^{\left\lceil c_{0} t^{2} k^{3}\right\rceil}\left(u^{(k)}(t, x)-1\right) d x\right|^{2}\right],
\end{aligned}
$$

where we used the stationarity in the last inequality. We now partition $[-L, L]$ into subintervals of size $L^{\prime \prime}:=\left\lceil c_{0} t^{2} k^{3}\right\rceil$ and call those subintervals $I_{i}$ 's. Once again, thanks to Lemma 2.3, we can have a collection of i.i.d. random variables $\left\{\int_{I_{i}}\left(u^{(k)}(t, x)-1\right) d x: i=1,3, \ldots\right\}$ so that

$$
\begin{aligned}
\operatorname{Var}\left(\int_{\Lambda_{L}} u^{(k)}(t, x) d x\right) & \geq \sum_{i=1}^{\left\lfloor L / 2 L^{\prime \prime}\right\rfloor} \operatorname{Var}\left(\int_{I_{2 i-1}} u^{(k)}(t, x) d x\right) \\
& =\left\lfloor\frac{L}{2 L^{\prime \prime}}\right\rfloor \cdot \mathbb{E}\left[\left|\int_{0}^{\left\lceil c_{0} t^{2} k^{3}\right\rceil}\left(u^{(k)}(t, x)-1\right) d x\right|^{2}\right] .
\end{aligned}
$$

Since $q \leq 2 L / L^{\prime}$ and $L^{\prime \prime} / L^{\prime} \rightarrow 0$ as $t \rightarrow \infty$, we show that the first term on the right hand side of (5.6) converges to 0 in $L^{2}(\Omega)$, which also implies the convergence in probability to 0 .

We now focus on the second term in (5.6). Here, we apply the Lyapunov criterion for the central limit theorem. From the localization argument, we know that $\left\{u^{(k)}(t, x), x \in \Lambda_{i}^{\prime}\right\}$ and $\left\{u^{(k)}(t, x), x \in \Lambda_{j}^{\prime}\right\}$ are independent if $i \neq j$. Thus, we only need to show that for any $\epsilon>0$,

$$
\limsup _{t \rightarrow \infty} \frac{\sum_{i \in \mathcal{I}} \mathbb{E}\left[\left|\int_{\Lambda_{i}^{\prime}}\left(u^{(k)}(t, x)-1\right) d x\right|^{2+\epsilon}\right]}{\left(\sum_{i \in \mathcal{I}} \operatorname{Var}\left(\int_{\Lambda_{i}^{\prime}} u^{(k)}(t, x) d x\right)\right)^{1+\epsilon / 2}}=0 .
$$

By Jensen's inequality, we obtain that

$$
\begin{aligned}
\sum_{i \in \mathcal{I}} \mathbb{E}\left[\left|\int_{\Lambda_{i}^{\prime}}\left(u^{(k)}(t, x)-1\right) d x\right|^{2+\epsilon}\right] & \leq C\left(\frac{L}{L^{\prime}}\right) \cdot\left(L^{\prime}\right)^{2+\epsilon} \exp \{(\bar{\gamma}(2+\epsilon)+o(1)) t\} \\
& \leq C \exp \left\{\left((1+\epsilon) \lambda^{\prime}+\lambda+\bar{\gamma}(2+\epsilon)+o(1)\right) t\right\}
\end{aligned}
$$

as $t \rightarrow \infty$. Once we use Lemma 5.1 with (5.3) (i.e. replace $L$ by $L^{\prime}$ in Lemma 5.1), we have

$$
\begin{aligned}
\left(\sum_{i \in \mathcal{I}} \operatorname{Var}\left(\int_{\Lambda_{i}^{\prime}} u^{(k)}(t, x) d x\right)\right)^{1+\epsilon / 2} & \geq C\left(\frac{L}{L^{\prime}}\right)^{1+\epsilon / 2}\left(2 L^{\prime} \int_{0}^{t} \xi(r) d r\right)^{1+\epsilon / 2} \\
& \geq C L^{1+\epsilon / 2} e^{(\underline{\gamma}(2)+o(1))(1+\epsilon / 2) t} \\
& =C \exp \left\{(\lambda+\underline{\gamma}(2)+o(1))\left(1+\frac{\epsilon}{2}\right) t\right\}
\end{aligned}
$$


Therefore, we have

$$
\begin{aligned}
& \frac{\sum_{i \in \mathcal{I}} \mathbb{E}\left[\left|\int_{\Lambda_{i}^{\prime}}\left(u^{(k)}(t, x)-1\right) d x\right|^{2+\epsilon}\right]}{\left(\sum_{i \in \mathcal{I}} \operatorname{Var}\left(\int_{\Lambda_{i}^{\prime}} u^{(k)}(t, x) d x\right)\right)^{1+\epsilon / 2}} \\
& \quad \leq C \exp \left\{\left((1+\epsilon) \lambda^{\prime}-\epsilon\left(\frac{\lambda}{2}-\frac{\bar{\gamma}(2+\epsilon)-\underline{\gamma}(2)}{\epsilon}+\frac{\underline{\gamma}(2)}{2}\right)+o(1)\right) t\right\} .
\end{aligned}
$$

Since $\lambda^{\prime}$ is arbitrary and $\lambda>\inf _{\epsilon>0}\left\{\frac{2(\bar{\gamma}(2+\epsilon)-\underline{\gamma}(2))}{\epsilon}-\underline{\gamma}(2)\right\}$, we prove (5.7), which completes the proof of (i) in Theorem 1.3.

For the proof of (ii), as in the proof of (i), it suffices to prove that, for a sufficiently large $k$ and for all $p \in(1,2]$,

$$
E\left[\left|\frac{\int_{\Lambda_{L}}\left(u^{(k)}(t, x)-1\right) d x}{\sqrt{\operatorname{Var}\left(\int_{\Lambda_{L}} u^{(k)}(t, x) d x\right)}}\right|^{p}\right] \rightarrow 0 \quad \text { as } t \rightarrow \infty
$$

provided that $0<\lambda<\frac{2 p}{2-p}\left(\frac{\gamma(2)}{2}-\frac{\bar{\gamma}(p)}{p}\right)$. Using (3.3) and Lemma 5.1, we have

$$
\mathbb{E}\left[\left|\frac{\int_{\Lambda_{L}}\left[u^{(k)}(t, x)-1\right] d x}{\sqrt{\operatorname{Var}\left(\int_{\Lambda_{L}} u^{(k)}(t, x) d x\right)}}\right|^{p}\right] \leq \exp \left\{\left(\lambda+\lambda^{\prime}(p-1)+\bar{\gamma}(p)-\frac{p}{2} \lambda-\frac{p}{2} \underline{\gamma}(2)+o(1)\right) t\right\},
$$

for all $\lambda^{\prime} \in(0, \lambda)$. Since $\lambda^{\prime}$ is arbitrary, as long as $0<\lambda<\frac{2 p}{2-p}\left(\frac{\gamma(2)}{2}-\frac{\bar{\gamma}(p)}{p}\right)$, the right hand side of (5.10) converges to 0 as $t \rightarrow \infty$. This completes the proof of (ii).

\section{Proof of Theorem 1.5}

In this section, we provide a proof of Theorem 1.5 (the quantitative central limit theorem) by following the proof of Theorem 1.1 in [18] where the authors showed the quantitative central limit theorem for the spatial average $\frac{1}{2 R} \int_{-R}^{R}(u(t, x)-1) d x$ as $R \rightarrow \infty$ for fixed $t>0$ by using the Malliavin calculus and Stein's method (see also [5, Corollary 2.7] where the authors consider $N^{-d} \int_{[0, N]^{d}}\left(u\left(t_{N}, x\right)-1\right) d x$ where $t_{N}=o(\log N)$ as $\left.N \rightarrow \infty\right)$. Although our proof is very similar to the proof of Theorem 1.1 in [18], we prefer to give a self-contained proof for completeness and also our proof indicates a precise length of the interval which guarantees the quantitative central limit theorem. Note that the method combining the Malliavin calculus and Stein's method used in $[5,18]$ does not require any of techniques from the previous sections such as a localization argument to get independence. We first introduce briefly some basic notations and facts about the Malliavin calculus and Stein's method for the sake of completeness (see Section 2 of [18] and also Chapter 5 by Nourdin and Peccati [24] for more details). 
Define $H:=L^{2}\left(\mathbb{R}_{+} \times \mathbb{R}\right)$ and the Wiener integral $h \mapsto W(h)$ as

$$
W(h)=\int_{0}^{\infty} \int_{\mathbb{R}} h(t, x) W(d t d x) \quad \text { for } h \in H .
$$

We denote by $D$ the Malliavin derivative operator and by $\delta$ the adjoint of the derivative operator $D$ given by the duality formula

$$
\mathbb{E}[\delta(u) F]=\mathbb{E}\left[\langle u, D F\rangle_{H}\right]
$$

for any $F$ in the Gaussian Sobolev space $\mathbb{D}^{1,2}$ and any $u \in L^{2}(\Omega ; H)$ in the domain of $\delta$, denoted by $\operatorname{Dom} \delta$. Note that any predictable random field $X$ which satisfies

$$
\int_{0}^{\infty} \int_{\mathbb{R}}\|X(s, y)\|_{2}^{2} d y d s<\infty
$$

belongs to Dom $\delta$ and $\delta(X)$ coincides with the Walsh integral. Here, we define $\|X\|_{k}:=$ $\left\{\mathbb{E}\left[|X|^{k}\right]\right\}^{1 / k}$ for a random variable $X$. Thus, we can write the solution $u(t, x)$ to $(1.1)$ as

$$
u(t, x)=1+\delta\left(p_{t-.}(x-*) u(\cdot, *)\right) .
$$

In addition, it is well-known that $u(t, x)$ belongs to $\mathbb{D}^{1, p}$ for any $p \geq 2$ for any $(t, x)$ and the Malliavin derivative of $u$ satisfies the following equation for a.e. $(s, y) \in(0, t) \times \mathbb{R}$

$$
\begin{aligned}
D_{s, y} u(t, x)= & p_{t-s}(x-y) \sigma(u(s, y)) \\
& +\int_{s}^{t} \int_{\mathbb{R}} p_{t-r}(x-z) \Sigma(r, z) D_{s, y} u(r, z) W(d r d z),
\end{aligned}
$$

where $\Sigma(r, z)$ is an adapted process, bounded by $\operatorname{Lip}_{\sigma}$. If $\sigma$ is continuously differentiable, then $\Sigma(r, z)=\sigma^{\prime}(u(r, z))$. From Lemma 4.2 in [4], we know that for all $0<\epsilon<1, t \geq s \geq r>0$, $k \geq 2$, and for all $x \in \mathbb{R}$,

$$
\left\|D_{r, z} u(s, y)\right\|_{k} \leq C_{1}(\sigma, \epsilon) p_{s-r}(y-z) \exp \left\{C_{2}(\sigma, \epsilon, k) t\right\}
$$

holds for a.e. $(r, z) \in(0, s) \times \mathbb{R}$ where

$$
C_{1}(\sigma, \epsilon):=\frac{8 \operatorname{Lip}_{\sigma}}{\epsilon^{3 / 2}} \quad \text { and } \quad C_{2}(\sigma, \epsilon, k):=\frac{2^{3} k^{2}\left(\operatorname{Lip}_{\sigma}\right)^{4}}{(1-\epsilon)^{4}} .
$$

Note that $C_{2}(\sigma, \epsilon, k)$ in (6.6) was obtained from the following definition in Lemma 4.2 of [4]:

$$
C_{2}(\sigma, \epsilon, k):=2 \zeta\left(\frac{a(\epsilon)}{k}\right)
$$

where, for all $x \geq 0$,

$$
a(\epsilon):=\frac{(1-\epsilon)^{2}}{2^{3 / 2}\left(\operatorname{Lip}_{\sigma}\right)^{2}}, \quad \zeta(x):=\rho^{-1}(x) \quad \text { and } \quad \rho(x):=\frac{1}{\pi} \int_{\mathbb{R}} \frac{1}{2 x+|\xi|^{2}} d \xi=\frac{1}{\sqrt{2 x}} .
$$

We now define the total variance distance between two random variables $F$ and $G$ by

$$
d_{T V}(F, G)=\sup _{B \in \mathcal{B}(\mathbb{R})}|\mathbb{P}[F \in B]-\mathbb{P}[G \in B]|,
$$

where $\mathcal{B}(\mathbb{R})$ is the Borel sigma field on $\mathbb{R}$. The next proposition which combines the Malliavin calculus and Stein's method is given in [18] (see also Nourdin-Peccati [24]). 
Proposition 6.1 (Proposition 2.2 in [18]). Let $F \in \mathbb{D}^{1,2}$ be given by $F=\delta(u)$ for some $u \in \operatorname{Dom} \delta$. Let $Z \sim N(0,1)$. If $\mathbb{E}\left[F^{2}\right]=1$, then we have

$$
d_{T V}(F, Z) \leq 2 \sqrt{\operatorname{Var}\langle D F, u\rangle_{H}} .
$$

We now provide a proof of Theorem 1.5 by following the proof of Theorem 1.1 of [18].

Proof of Theorem 1.5. Let $L:=L(t):=e^{\lambda t}$ for $\lambda>0$. Define

$$
\sigma_{\lambda}(t):=\sqrt{\operatorname{Var}\left(\int_{\Lambda_{L}} u(t, x) d x\right)} \text { and } F_{\lambda}(t):=\frac{1}{\sigma_{\lambda}(t)}\left(\int_{\Lambda_{L}}[u(t, x)-1] d x\right) .
$$

By stochastic Fubini's theorem and (1.2), we can write

$$
F_{\lambda}(t)=\int_{0}^{t} \int_{\mathbb{R}}\left(\frac{1}{\sigma_{\lambda}(t)} \int_{\Lambda_{L}} p_{t-s}(x-y) \sigma(u(s, y)) d x\right) W(d s d y) .
$$

Thus, for any fixed $t \geq 0, F_{\lambda}(t)=\delta\left(v_{\lambda}\right)$ where

$$
v_{\lambda}(s, y):=v_{\lambda}^{(t)}(s, y):=1_{[0, t]}(s) \frac{1}{\sigma_{\lambda}(t)} \int_{\Lambda_{L}} p_{t-s}(x-y) \sigma(u(s, y)) d x .
$$

Since the derivative operator $D$ can be interchanged with Lebesgue integration, we have

$$
D_{s, y} F_{\lambda}(t)=1_{[0, t]} \frac{1}{\sigma_{\lambda}(t)} \int_{\Lambda_{L}} D_{s, y} u(t, x) d x
$$

Therefore, we get

$$
\left\langle D F_{\lambda}(t), v_{\lambda}\right\rangle_{H}=\frac{1}{\sigma_{\lambda}^{2}(t)} \int_{0}^{t} \int_{\mathbb{R}} \int_{\Lambda_{L}} \int_{\Lambda_{L}} p_{t-s}(x-y) \sigma(u(s, y)) D_{s, y} u\left(t, x^{\prime}\right) d x d x^{\prime} d y d s .
$$

Our goal is to find $\lambda$ which guarantees $\operatorname{Var}\left(\left\langle D F_{\lambda}(t), v_{\lambda}\right\rangle_{H}\right) \rightarrow 0$ as $t \rightarrow \infty$. From (6.4), we can compute

$$
\begin{aligned}
\left\langle D F_{\lambda}(t), v_{\lambda}\right\rangle_{H}= & \frac{1}{\sigma_{\lambda}^{2}(t)} \int_{0}^{t} \int_{\mathbb{R}}\left(\int_{\Lambda_{L}} p_{t-s}(x-y) d x\right)^{2} \sigma^{2}(u(s, y)) d y d s \\
& +\frac{1}{\sigma_{\lambda}^{2}(t)} \int_{0}^{t} \int_{\mathbb{R}} \int_{\Lambda_{L}} \int_{\Lambda_{L}} p_{t-s}(x-y) \sigma(u(s, y)) \\
& \times\left(\int_{s}^{t} \int_{\mathbb{R}} p_{t-r}(\tilde{x}-z) \Sigma(r, z) D_{s, y} u(r, z) W(d r d z)\right) d x d \tilde{x} d y d s .
\end{aligned}
$$

Using Minkowski's inequality for integrals, we can see that

$$
\sqrt{\operatorname{Var}\left(\left\langle D F_{\lambda}(t), v_{\lambda}\right\rangle_{H}\right)} \leq B_{1}+B_{2},
$$

where

$$
\begin{aligned}
B_{1}:= & \frac{1}{\sigma_{\lambda}^{2}(t)} \int_{0}^{t}\left(\int_{\mathbb{R}^{2}}\left(\int_{\Lambda_{L}} p_{t-s}(x-y) d x\right)^{2}\left(\int_{\Lambda_{L}} p_{t-s}\left(x^{\prime}-y-\right) d x^{\prime}\right)^{2}\right. \\
& \left.\times \operatorname{Cov}\left(\sigma^{2}(u(s, y)), \sigma^{2}\left(u\left(s, y^{\prime}\right)\right)\right) d y d y^{\prime}\right)^{\frac{1}{2}} d s
\end{aligned}
$$


and

$$
\begin{aligned}
B_{2}:= & \frac{1}{\sigma_{\lambda}^{2}(t)} \int_{0}^{t}\left(\int_{\mathbb{R}^{2}} \int_{\left(\Lambda_{L}\right)^{4}} p_{t-s}(x-y) p_{t-s}\left(x^{\prime}-y^{\prime}\right) \int_{s}^{t} \int_{\mathbb{R}} p_{t-r}(\tilde{x}-z) p_{t-r}\left(\tilde{x}^{\prime}-z\right)\right. \\
& \left.\times \mathbb{E}\left[\sigma(u(s, y)) \sigma\left(u\left(s, y^{\prime}\right)\right) \Sigma^{2}(r, z) D_{s, y} u(r, z) D_{s, y^{\prime}} u(r, z)\right] d z d r d x d x^{\prime} d \tilde{x} d \tilde{x}^{\prime} d y d y^{\prime}\right)^{\frac{1}{2}} d s .
\end{aligned}
$$

We first estimate $B_{1}$. Applying the Clark-Ocone formula to $\sigma^{2}(u(s, y))$ as in [18], we have

$\operatorname{Cov}\left(\sigma^{2}(u(s, y)), \sigma^{2}\left(u\left(s, y^{\prime}\right)\right)\right)=\int_{0}^{s} \int_{\mathbb{R}} \mathbb{E}\left[\mathbb{E}\left[D_{r, z}\left(\sigma^{2}(u(s, y))\right) \mid \mathcal{F}_{r}\right] \mathbb{E}\left[D_{r, z}\left(\sigma^{2}\left(u\left(s, y^{\prime}\right)\right)\right) \mid \mathcal{F}_{r}\right]\right] d z d r$.

In addition, as it was shown in [18], we have

$$
\left\|\mathbb{E}\left[D_{r, z}\left(\sigma^{2}(u(s, y))\right) \mid \mathcal{F}_{r}\right]\right\|_{2} \leq C K_{4}(t)\left\|D_{r, z} u(s, y)\right\|_{4},
$$

where $C$ is a positive constant which depends only on $\operatorname{Lip}_{\sigma}$ and $K_{p}(t)$ is defined as

$$
K_{p}(t)=\sup _{0 \leq s \leq t} \sup _{y \in \mathbb{R}}\|\sigma(u(s, y))\|_{p} \quad \text { for } p \geq 2 .
$$

Since $|\sigma(u)| \leq \operatorname{Lip}_{\sigma}|u|$, we have

$$
K_{p}(t) \leq \operatorname{Lip}_{\sigma} e^{\left(\frac{\bar{\gamma}(p)}{p}+o(1)\right) t},
$$

as $t \rightarrow \infty$. Let $0<\epsilon<1$ be fixed and $C_{2}=C_{2}(\sigma, \epsilon, 4)$ in (6.6). We denote by $C$ a constant that depends on $\operatorname{Lip}_{\sigma}$ and can be changed line by line. Using (6.5), we have

$$
\begin{aligned}
\left|\operatorname{Cov}\left(\sigma^{2}(u(s, y)), \sigma^{2}\left(u\left(s, y^{\prime}\right)\right)\right)\right| & \leq C K_{4}^{2}(t) \int_{0}^{s} \int_{\mathbb{R}}\left\|D_{r, z} u(s, y)\right\|_{4}\left\|D_{r, z} u\left(s, y^{\prime}\right)\right\|_{4} d z d r \\
& \leq C e^{\left(2 C_{2}+\frac{\bar{\gamma}(4)}{2}+o(1)\right) t} \int_{0}^{s} \int_{\mathbb{R}} p_{s-r}(y-z) p_{s-r}\left(y^{\prime}-z\right) d z d r \\
& =C e^{\left(2 C_{2}+\frac{\bar{\gamma}(4)}{2}+o(1)\right) t} \int_{0}^{s} p_{2 s-2 r}\left(y-y^{\prime}\right) d r .
\end{aligned}
$$

Moreover, Lemma 5.1 implies that as $t \rightarrow \infty$

$$
\sigma_{\lambda}^{2}(t) \geq L(t) \int_{0}^{t} \xi(r) d r \geq e^{(\lambda+\underline{\gamma}(2)+o(1)) t} .
$$

We may also follow [18] (i.e. replace $R$ by $L=e^{\lambda t}$ ) to see that

$$
\begin{aligned}
& \int_{0}^{t}\left(\int_{\mathbb{R}^{2}}\left(\int_{\Lambda_{L}} p_{t-s}(x-y) d x\right)^{2}\left(\int_{\Lambda_{L}} p_{t-s}\left(x^{\prime}-y-\right) d x^{\prime}\right)^{2} \int_{0}^{s} p_{2 s-2 r}\left(y-y^{\prime}\right) d r d y d y^{\prime}\right)^{\frac{1}{2}} d s \\
& \leq C \int_{0}^{t}\left(\int_{0}^{s} \int_{\left(\Lambda_{L}\right)^{2}} p_{2 t-2 r}\left(x-x^{\prime}\right) d x d x^{\prime} d r\right)^{\frac{1}{2}} d s \\
& \leq C t^{3 / 2}\left|\Lambda_{L}\right|^{1 / 2}
\end{aligned}
$$


Combining things together, we have

$$
B_{1} \leq C \exp \left\{-\left(\frac{1}{2} \lambda+\underline{\gamma}(2)-2 C_{2}-\frac{\bar{\gamma}(4)}{2}+o(1)\right) t\right\}
$$

as $t \rightarrow \infty$.

Now we estimate $B_{2}$. By Hölder's inequality,

$$
\begin{aligned}
& \mathbb{E}\left[\sigma(u(s, y)) \sigma\left(u\left(s, y^{\prime}\right)\right) \Sigma^{2}(r, z) D_{s, y} u(r, z) D_{s, z^{\prime}} u(r, z)\right] \\
& \quad \leq C K_{4}^{2}(t)\left\|D_{s, y} u(r, z)\right\|_{4}\left\|D_{s, y^{\prime}} u(r, z)\right\|_{4} .
\end{aligned}
$$

Again using (6.5), we have

$$
\begin{aligned}
B_{2} \leq C \frac{e^{\left(2 C_{2}+\frac{\bar{\gamma}(4)}{2}+o(1)\right) t}}{e^{(\lambda+\underline{\gamma}(2)+o(1)) t}} & \int_{0}^{t}\left(\int_{\mathbb{R}^{2}} \int_{\left(\Lambda_{L}\right)^{4}} \int_{s}^{t} \int_{\mathbb{R}} p_{t-s}(x-y) p_{t-s}\left(x^{\prime}-y^{\prime}\right) p_{t-r}(\tilde{x}-z)\right. \\
& \left.\times p_{t-r}\left(\tilde{x}^{\prime}-z\right) p_{r-s}(z-y) p_{r-s}\left(z-y^{\prime}\right) d z d r d x d x^{\prime} d \tilde{x} d \tilde{x}^{\prime} d y d y^{\prime}\right)^{\frac{1}{2}} d s .
\end{aligned}
$$

Following [18], i.e., replacing $R$ by $L=e^{\lambda t}$, we have

$$
\begin{aligned}
& \int_{0}^{t}\left(\int_{\mathbb{R}^{2}} \int_{\left(\Lambda_{L}\right)^{4}} \int_{s}^{t} \int_{\mathbb{R}} p_{t-s}(x-y) p_{t-s}\left(x^{\prime}-y^{\prime}\right) p_{t-r}(\tilde{x}-z) p_{t-r}\left(\tilde{x}^{\prime}-z\right) p_{r-s}(z-y)\right. \\
& \left.\quad \times p_{r-s}\left(z-y^{\prime}\right) d z d r d x d x^{\prime} d \tilde{x} d \tilde{x}^{\prime} d y d y^{\prime}\right)^{\frac{1}{2}} d s \\
& \leq \int_{0}^{t}\left(\int_{\left(\Lambda_{L}\right)^{2}} \int_{s}^{t} p_{2 t+2 r-4 s}\left(x-x^{\prime}\right) d r d x d x^{\prime}\right)^{\frac{1}{2}} d s \\
& \leq C t^{3 / 2}\left|\Lambda_{L}\right|^{1 / 2}
\end{aligned}
$$

Hence, we obtain

$$
B_{2} \leq C \exp \left\{-\left(\frac{1}{2} \lambda+\underline{\gamma}(2)-2 C_{2}-\frac{\bar{\gamma}(4)}{2}+o(1)\right) t\right\}
$$

as $t \rightarrow \infty$. We note that $C_{2}=C_{2}(\sigma, \epsilon, 4)=2^{7} \operatorname{Lip}_{\sigma}^{4} /(1-\epsilon)^{4}$ strictly decreases as $\epsilon \rightarrow 0$. Therefore, if we redefine $C_{2}=2^{7} \operatorname{Lip}_{\sigma}^{4}$ and choose $\lambda$ so that

$$
\lambda>2{ }^{9} \operatorname{Lip}_{\sigma}^{4}+\bar{\gamma}(4)-2 \underline{\gamma}(2)
$$

then this completes the proof by taking $\epsilon$ close to 0 .

Remark 6.2. It is known that in the case of $\sigma(u)=\operatorname{Lip}_{\sigma} u, \bar{\gamma}(2)=\gamma(2)=\operatorname{Lip}_{\sigma}^{4} / 4$ (see Corollary 2.8 in [15]). Thus, the condition on $\lambda$ in (6.11) becomes

$$
\lambda>\left(2^{11}-2\right) \gamma(2)+\gamma(4) .
$$

Since we know that $\gamma(p)=\operatorname{Lip}_{\sigma}^{4} p\left(p^{2}-1\right) / 24$ for all $p>0$ (see [17]), we can see that $\left(2^{11}-\right.$ 2) $\gamma(2)+\gamma(4)>2 \gamma^{\prime}(2)-\gamma(2)$. For the general $\sigma$, one may use the moment comparison principle $([7,19])$ to get the bound on $\lambda$. 


\section{Acknowledgments}

We appreciate Davar Khoshnevisan for stimulating discussions and suggestions. We also thank Carl Mueller and Mohammud Foondun for useful discussions.

\section{References}

[1] G. Ben Arous, S. A. Molchanov and A.F. Ramirez. Transition from the annealed to the quenched asymptotics for a random walk on random obstacles, Ann. Probab. 33 (2005), no. 6, 2149-2187.

[2] R. A. Carmona and S. A. Molchanov. Parabolic Anderson Problem and Intermittency, Memoires of the Amer. Math. Soc. 108, American Mathematical Society, Rhode Island, 1994.

[3] L. Chen, D. Khoshnevisan, D. Nualart and F. Pu. Spatial ergodicity for SPDEs via Poincaré-type inequalities, submitted, 2019, preprint available at https://arxiv.org/abs/1907.11553.

[4] L. Chen, D. Khoshnevisan, D. Nualart and F. Pu. Poincaré inequality, and central limit theorems for parabolic stochastic partial differential equations, submitted, 2019, preprint available at https://arxiv.org/abs/1912.01482.

[5] L. Chen, D. Khoshnevisan, D. Nualart and F. Pu. Central limit theorems for spatial averges of the stochastic heat equation via Malliavin-Stein's method, submitted, 2020, preprint available at https://arxiv.org/abs/2008.02408.

[6] L. Chen and K. Kim. On comparison principle and strict positivity of solutions to the nonlinear stochastic fractional heat equations. Ann. Inst. Henri Poincaré Probab. Stat., 53 (2017), no. 1, $358-388$.

[7] L. Chen and K. Kim. Stochastic comparisons for stochastic heat equation, submitted, 2019, preprint available at https://arxiv.org/abs/1912.05350.

[8] X. Chen. Precise intermittency for the parabolic Anderson equation with an (1+1)-dimensional time-space white noise, Ann. Inst. Henri Poincaré Probab. Stat. 51(4) (2015), 1486-1499.

[9] D. Conus, J. Mathew and D. Khoshnevisan. On the chaotic character of the stochastic heat equation, before the onset of intermittency, Ann. Probab. 41(3B) (2013), 2225-2260.

[10] M. Cranston and S. Molchanov. Quenched to annealed transition in the parabolic Anderson problem, Probab. Th. Rel. Fields 138 (2007), no. 1-2, 177-193.

[11] M. Cranston, T. Mountford, T. Shiga. Lyapunov exponents for the parabolic Anderson model, Acta Math. Univ. Comenian. 71 (2002), no. 2, 163-188.

[12] R. C. Dalang. Extending the martingale measure stochastic integral with applications to spatially homogeneous s.p.d.e.'s, Electron. J. Probab. 4(6) (1999) 29 pp.

[13] S. Das and L.C. Tsai. Fractional moments of the Stochastic Heat Equation, submitted, 2019, preprint available at https://arxiv.org/abs/1910.09271.

[14] S. W. Dharmadhikari and K. Jogdeo. Bounds on moments of certain random variables, Ann. Math. Stat. 40(4) (1969), 1506-1509

[15] M. Foondun and D. Khoshnevisan. Intermittence and nonlinear stochastic partial differential equations, Electr. J. Probab. 14(2) (2009) 548-568.

[16] J. Gärtner and A. Schnitzler. Stable limit laws for the parabolic Anderson model between quenched and annealed behaviour. Ann. Inst. Henri Poincaré Probab. Stat. 51(1), (2015) 194-206.

[17] P. Ghosal and Y. Lin. Lyapunov exponents of the SHE for general initial data, submitted, 2020 preprint available at https://arxiv.org/abs/2007.06505.

[18] J. Huang, D. Nualart and L. Viitasaari. A central limit theorem for the stochastic heat equation, Stoch. Process. Their Appl. 130 (2020), no. 12, 7170-7184. 
[19] M. Joseph, D. Khoshnevisan and C. Mueller. Strong invariance and noise-comparison principles, Ann. Probab. 45 (2017), no. 1, 377-403.

[20] D. Khoshnevisan. Analysis of Stochastic Partial Differential Equations, CBMS Regional Conference Series in Mathematics, 119. American Mathematical Society, Providence, RI, 2014.

[21] D. Khoshnevisan, K. Kim and Y, Xiao. Intermittency and multifractality: A case study via parabolic stochastic PDEs, Ann. Probab. 45 (2017), no. 6A, 3697-3751

[22] D. Khoshnevisan, K. Kim and Y, Xiao. Macroscopic Multifractal Analysis of Parabolic Stochastic PDEs, Commun. Math. Phys. 360 (2018), 307-346.

[23] C. Mueller. On the support of solutions to the heat equation with noise. Stochastics, 37 (1991), 225-246.

[24] I. Nourdin and G. Peccati. Normal approximations with Malliavin calculus. From Stein's method to universality. Cambridge University Press, Cambridge, UK., 2012.

[25] B. von Bahr and C-G. Esseen. Inequalities for the $r$ th Absolute Moment of a Sum of Random Variables, $1 \leqq r \leqq 2$. Ann. Math. Statist. 36 (1965), no. 1, 299-303.

[26] J. B. Walsh. An Introduction to Stochastic Partial Differential Equations, in: École d'été de probabilités de Saint-Flour, XIV-1984, 265-439, Lecture Notes in Math., vol. 1180, Springer, Berlin, 1986.

Kunwoo Kim [kunwookim@postech.ac.kr]

Pohang University of Science and Technology (POSTECH), Pohang, Gyeongbuk, South Korea

Jaeyun Yi [stork@postech.ac.kr]

Pohang University of Science and Technology (POSTECH), Pohang, Gyeongbuk, South Korea 